\title{
Testing whether the lifted group splits
}

\author{
Rok Požar* \\ Faculty of Mathematics, Natural Sciences and Information Technologies, \\ University of Primorska, Glagoljaška 8, 6000 Koper, Slovenia
}

Received 8 July 2014, accepted 22 June 2015, published online 1 October 2015

\begin{abstract}
Let a group of automorphisms lift along a regular covering projection of connected graphs given combinatorially by means of voltages. The data that determine the lifted group and its action are then conveniently encoded in terms of voltages as well. Along these lines, an algorithm for testing whether the lifted group is a split extension of the group of covering transformations has recently been proposed in the case when the group of covering transformations is solvable. It consists of decomposing the covering into a series of coverings with elementary abelian groups of covering transformations, and inductively solving the problem at every elementary abelian step. Although the explicit construction of the lifted group is not needed, it still involves time and space consuming constructions of certain subgroups in the lifted group at every step except at the final one.

In this paper, an improved version that completely avoids such constructions is presented. From voltage distribution we first compute the weak action and the factor set that determine the lifted group, and we then carry out the test by extracting the necessary information only from the corresponding weak actions and factor sets at every step. An experimental comparison is made against the previous version.
\end{abstract}

Keywords: Algorithm, graph, group extension, lifting automorphisms, regular covering projection, voltages.

Math. Subj. Class.: 05C50, 05E18, 20B40, 20B25, 20K35, 57M10

\section{Introduction}

Group extensions arising from lifting groups of automorphisms along regular graph coverings play a significant role in analyzing symmetry properties of graphs; see, for example, $[5,6,9,10,13,16,19]$. One therefore frequently needs to answer questions regarding structural properties of such extensions.

\footnotetext{
*This work is supported in part by the Slovenian Research Agency (research program P1-0285).

E-mail address: pozar.rok@gmail.com (Rok Požar)
} 
Specifically, let a group $G$ of automorphisms of a graph $X$ lift along a regular covering projection $\wp: \tilde{X} \rightarrow X$ to a group $\tilde{G}$ of automorphisms of the covering graph $\tilde{X}$. Then the lifted group $\tilde{G}$ is an extension of the group of covering transformations $\mathrm{CT}(\wp)$ by $G$. Often, all of the data about the lifted group and its action are conveniently encoded on $X$ by means of voltages that determine $\wp$. In such a situation we can always reconstruct $\tilde{G}$ as a permutation group acting on $\tilde{X}$, and then apply the known algorithms for permutation groups in order to investigate its structure. However, taking into account complexity issues, this reconstruction is expensive whenever $\mathrm{CT}(\wp)$ is large. Instead, we wish to reduce the investigation of structural properties of $\tilde{G}$ to the study of voltage distribution on $X$. A natural question of interest is then the following: for a group $G$ that lifts along $\wp$ given by means of voltages, is the lifted group $\tilde{G}$ a split extension of $\mathrm{CT}(\wp)$ by $G$ ?

There are efficient algorithms in computational group theory for testing whether a given group extension splits (see, for example, [3] and [8, Chapters 7 and 8]), and these functions have also been implemented in MAGMA [1]. Unfortunately, the algorithms as well as the implementations address the case when extensions are input as permutation groups.

In [15], an algorithm for testing whether the lifted group $\tilde{G}$ splits is described in the case when $\mathrm{CT}(\wp)$ is (elementary) abelian. It is based on extracting all the necessary information about $\tilde{G}$ from voltage distribution, rather then explicitly constructing $\tilde{G}$ as a permutation group.

This idea is taken further in [17] to deal with the case of a solvable CT $(\wp)$. The algorithm consists of decomposing $\wp$ into a series of regular covering projections with elementary abelian groups of covering transformations, and inductively applying the algorithm from [15] at every elementary abelian step. Although the explicit construction of $\tilde{G}$ is not needed, the algorithm still involves time and space consuming constructions of certain subgroups isomorphic to $G$ in the lifted group at (possibly) every step except at the finale one.

In this paper, we improve the algorithm from [17] by avoiding such constructions entirely. The approach is based on the fact that a group extension can be recaptured by have it written as a crossed product extension in terms of the corresponding weak action and a factor set. As a first step we compute the weak action and the factor set corresponding to $\tilde{G}$ from voltage distribution. At each step, we then carry out our test by extracting all the necessary information only from the corresponding weak actions and the factor sets.

The paper is organized as follows. In Section 2 we review some preliminary concepts about regular graph coverings and lifting automorphisms as well as group extensions. In Section 3 we discuss the problem of testing whether an extension splits in terms of weak actions and factor sets. In Section 4 we then propose an improved algorithm for testing whether the lifted group splits. Finally, we evaluate the performance of our algorithm in comparison with the previous version [17] in Section 5. Experimental results confirm the effectiveness of the improvements made.

\section{Preliminaries}

We begin with a review of some basic concepts in order to fix the notation and terminology.

\subsection{Regular graph covers and lifts of automorphism}

Throughout the paper, graphs are finite, simple and undirected. For a graph $X$ we denote by $V(X), A(X)$ its vertex and arc set, respectively. The full automorphism group of $X$ is 
denoted by $\operatorname{Aut}(X)$. For a detailed treatment of graph coverings and lifting automorphism we refer the reader to $[7,12,14]$.

A surjective graph homomorphism $\wp: \tilde{X} \rightarrow X$ is called a regular covering projection if there exists a semiregular subgroup $S_{\wp}$ of $\operatorname{Aut}(\tilde{X})$ such that its vertex orbits coincide with the vertex fibres $\wp^{-1}(v), v \in V(X)$. In this setting we call $X$ a base graph, and $\tilde{X}$ a covering graph (or a cover). Regular covering projections $\wp: \tilde{X} \rightarrow X$ and $\wp^{\prime}: \tilde{X}^{\prime} \rightarrow X$ are equivalent if there exists a graph isomorphism $\tilde{g}: \tilde{X} \rightarrow \tilde{X}^{\prime}$ such that $\wp=\tilde{g} \wp^{\prime}$.

An automorphism $g \in \operatorname{Aut}(X)$ lifts along $\wp: \tilde{X} \rightarrow X$ if there exists an automorphism $\tilde{g} \in \operatorname{Aut}(\tilde{X})$, called a lift of $g$, such that $\tilde{g} \wp=\wp g$. A group $G \leq \operatorname{Aut}(X)$ lifts if each $g \in G$ lifts. The collection of all lifts of all elements in $G$ forms a subgroup $G \leq \operatorname{Aut}(\tilde{X})$, called the lift of $G$ or the lifted group. In particular, the lift of the trivial group, denoted by $\mathrm{CT}(\wp)$, is known as the group of covering transformations. If $\mathrm{CT}(\wp)$ is an elementary abelian or a solvable group, the regular covering projection $\wp$ is called elementary abelian or solvable, respectively. Observe that $\mathrm{CT}(\wp)$ is a normal subgroup of $\tilde{G}$ and that $\tilde{G} / \mathrm{CT}(\wp) \cong G$, so $\tilde{G}$ is an extension of $\mathrm{CT}(\wp)$ by $G$.

Regular covering projections can be grasped combinatorially as follows. Let $N$ be a (finite) group. Define a voltage function $\zeta: A(X) \rightarrow N$ such that $\zeta\left(v_{2}, v_{1}\right)=\left(\zeta\left(v_{1}, v_{2}\right)\right)^{-1}$ for each $\left(v_{1}, v_{2}\right) \in A(X)$; that is, a function assigning mutually inverse elements in $N$ to mutually inverse arcs in $X$. We call $N$ the voltage group, while the values of $\zeta$ are called voltages. Further, construct the derived graph $X \times{ }_{\zeta} N$ with vertex set $V(X) \times N$ and adjacency relation $\left(v_{1}, n\right) \sim\left(v_{2}, n \zeta\left(v_{1}, v_{2}\right)\right)$ whenever $v_{1} \sim v_{2}$. The projection

$$
\wp_{\zeta}: X \times_{\zeta} N \rightarrow X, \quad(v, n) \mapsto v,
$$

is then the derived regular covering projection, where the required semiregular subgroup $S_{\wp_{\zeta}}$ of $\operatorname{Aut}\left(X \times_{\zeta} N\right)$ arises from the action of $N$ on the second coordinate by left multiplication on itself. Conversely, with any regular covering projection $\wp: \tilde{X} \rightarrow X$ there is an associated voltage function $\zeta$ on $X$ such that the derived covering projection $\wp_{\zeta}$ is equivalent to $\wp$. Since both graphs $\tilde{X}$ and $X$ are connected, the voltage function $\zeta$ associated with the projection $\wp$ is valued in $N \cong \mathrm{CT}(\wp)$ (viewed as an abstract group).

The fact that an automorphism lifts along a projection $\wp$ if and only if it lifts along along any covering projection equivalent to $\wp$ allows us to study lifts of automorphisms combinatorially in terms of voltage functions. Let $\zeta: A(X) \rightarrow N$ be a voltage function associated with a regular covering projection $\wp: \tilde{X} \rightarrow X$ of connected graphs. We note that $\zeta$ can be naturally extended to walks: if $W=v_{1} v_{2} \cdots v_{n-1} v_{n}$ is a walk in $X$, then $\zeta W=\zeta\left(v_{1}, v_{2}\right) \cdots \zeta\left(v_{n-1}, v_{n}\right)$. By the basic lifting lemma, see [12, 14], $g \in \operatorname{Aut}(X)$ lifts along $\wp$ if and only if there exists an automorphism $g^{\# v}$ of $N$ such that

$$
g^{\# v}(\zeta W)=\zeta g(W)
$$

for all closed walks $W$ in $X$ rooted at a fixed vertex $v$. Of course, if $g$ lifts, $g^{\# v}$ is uniquely determined by a map $\zeta W^{*} \mapsto \zeta g\left(W^{*}\right)$, where $W^{*}$ ranges over all fundamental closed walks in $X$ rooted at $v$.

\subsection{Group extensions}

A group $E$ is called a (group) extension of a group $N$ by a group $G$ if there is a short exact sequence

$$
1 \rightarrow N \stackrel{i}{\rightarrow} E \stackrel{q}{\rightarrow} G \rightarrow 1 .
$$


It is called a split extension if there is a homomorphism $j: G \rightarrow E$ with $q j=\mathrm{id}$. In particular, the group $E$ having a normal subgroup $N$ is an extension of $N$ by $E / N$, and it is a split extension if there is a transversal of $N$ in $E$ - a system of representatives in $E$ of cosets of $N$ in $E$ - that forms a group. Such a group is called a complement of $N$ in $E$. Group extensions $E$ and $E^{\prime}$ of $N$ by $G$ are equivalent if there exists an isomorphism $\alpha: E \rightarrow E^{\prime}$ such that the diagram

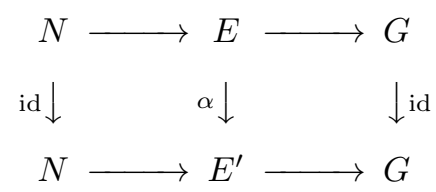

is commutative. Of course, if $E$ and $E^{\prime}$ are equivalent extensions, then $E$ is split if and only if $E^{\prime}$ is split.

Suppose that the group $E$ has a normal subgroup $N$. All of the data that determine the group operation in $E$ can be, up to equivalence of extensions, given in terms of $N$ and $G=E / N$. The approach is known and goes back to Schreier [11]. For each $g \in G$ fix a coset representative $\bar{g}$ in $E$ such that $\bar{g} N=g$. Since $N$ is normal, the element $\bar{g}$ gives rise to an automorphism $g^{\#}$ of $N$ defined by $g^{\#}(n)=\bar{g} n \bar{g}^{-1}$. Clearly, this definition depends on the choice of $\bar{g}$, and hence the function

$$
\#: G \rightarrow \operatorname{Aut}(N), \quad g \mapsto g^{\#},
$$

called a weak action, is not a group homomorphism in general. Further, the fact that the elements $\{\bar{g} \mid g \in G\}$ form a transversal of $N$ in $E$ implies that for any $g_{1}, g_{2} \in G$ we have $\overline{g_{1}} \overline{g_{2}}=\mathcal{F}\left(g_{1}, g_{2}\right) \overline{g_{1} g_{2}}$ for some unique $\mathcal{F}\left(g_{1}, g_{2}\right) \in N$. The function

$$
\mathcal{F}: G \times G \rightarrow N, \quad\left(g_{1}, g_{2}\right) \mapsto \overline{g_{1}} \bar{g}_{2}{\overline{g_{1} g_{2}}}^{-1},
$$

for this choice of coset representatives is called a factor set. It is natural to choose $\overline{1}=1$. Then $\mathcal{F}(1,1)=1$, and such a factor set is called normalized. This will be our standard assumption without loss of generality. The weak action \# and the factor set $\mathcal{F}$ defined above determine a group operation on the set $N \times G$; namely, $N \times G$ becomes a group, denoted by $N \operatorname{ext}_{\#, \mathcal{F}} G$, under the multiplication

$$
\left(n_{1}, g_{1}\right) *\left(n_{2}, g_{2}\right)=\left(n_{1} g_{1}^{\#}\left(n_{2}\right) \mathcal{F}\left(g_{1}, g_{2}\right), g_{1} g_{2}\right) .
$$

In fact, $N \operatorname{ext}_{\#, \mathcal{F}} G$ is an extension of $N$ by $G$, called the crossed product extension, and is equivalent to $E$. More precisely, there exists an isomorphism

$$
N \operatorname{ext}_{\#, \mathcal{F}} G \rightarrow E, \quad(n, g) \mapsto n \bar{g},
$$

mapping $N \times 1$ onto $N$ and $1 \times G$ onto the transversal $\{\bar{g} \mid g \in G\}$.

\section{Testing whether an extension splits}

Let $N$ be a normal subgroup of a finite group $E$, and let $G=E / N$. We first briefly describe a general strategy for testing whether $E$ is a split extension of $N$ by $G$. In principal we follow [3] and [8, Chapters 7 and 8], however, for reasons that will become apparent in Section 4, we extract the necessary information from the crossed product extension $N \operatorname{ext}_{\#, \mathcal{F}} G$ that reconstructs $E$. 
Let $G=\langle S \mid R\rangle$ be a finite presentation of $G$, where $S=\left\{g_{1}, \ldots, g_{n}\right\}$ is a set of generators and $R=\left\{r_{1}\left(g_{1}, \ldots, g_{n}\right), \ldots, r_{m}\left(g_{1}, \ldots, g_{n}\right)\right\}$ is a set of relators - that is, a set of words in generators representing the identity element in $G$. We note that neither \# is determined uniquely by its values $g_{i}^{\#}$ for $g_{i} \in S$, nor $\mathcal{F}$ is determined uniquely by its values $\mathcal{F}\left(g_{i}, g_{j}\right)$ for $g_{i}, g_{j} \in S$. But this is not a problem; as we shall see in (3.2) and (3.3) below, it is enough to only know the images $g_{i}^{\#}$ of the generators $g_{i} \in S$ under \#, along with some particular images under $\mathcal{F}$.

A general transversal of $N \times 1$ in $N \operatorname{ext}_{\#, \mathcal{F}} G$ has the form $\{(\delta(g), g) \mid g \in G\}$ for a function $\delta: G \rightarrow N$. The same function also determines a transversal of $N$ in $E$, namely $\{\delta(g) \bar{g} \mid g \in G\}$, where $\{\bar{g} \mid g \in G\}$ is a transversal of $N$ in $E$ giving rise to the isomorphism $N \operatorname{ext}_{\#, \mathcal{F}} G \rightarrow E,(n, g) \mapsto n \bar{g}$, see (2.2).

As it is known, $E$ splits if and only if there exist coset representatives in $E$ of the generators of $G$ satisfying the defining relators of $G$. More precisely, if and only if, for each $g_{i}$ in $S$, there exists an element $\overline{g_{i}}$ in $E$ such that $\overline{g_{i}} N=g_{i}$ and that, for each relator $r_{j}$ in $R$, the word $r_{j}\left(\overline{g_{1}}, \ldots, \overline{g_{n}}\right)$ obtained from $r_{j}$ by replacing each $g_{i}$ by $\overline{g_{i}}$ whenever it

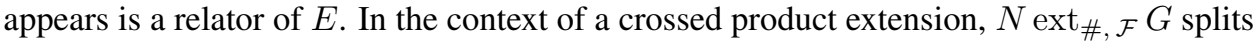
if and only if there exists a function $\delta: S \rightarrow N$ such that, for all $r_{j} \in R$,

$$
r_{j}\left(\left(\delta\left(g_{1}\right), g_{1}\right), \ldots,\left(\delta\left(g_{n}\right), g_{n}\right)\right)=(1,1)
$$

in $N \operatorname{ext}_{\#, \mathcal{F}} G$. Then the function $\delta$ defined on the generators extends to $\delta: G \rightarrow N$, and a complement is generated by the set $\left\{\left(\delta\left(g_{1}\right), g_{1}\right), \ldots,\left(\delta\left(g_{n}\right), g_{n}\right)\right\}$.

Let us now rewrite (3.1) explicitly in terms of the weak action and the factor set. Suppose $r_{j}=g_{j_{1}} \cdots g_{j_{t}} \in R$. Taking into account the multiplication rule (2.1) in $N \operatorname{ext}_{\#, \mathcal{F}} G$, denoted by $*$, and considering $(\delta(g), g)$ as $(\delta(g), 1) *(1, g)$, the condition (3.1) becomes

$$
\left(\delta\left(g_{j_{1}}\right) \prod_{k=2}^{t} g_{j_{1}}^{\#} \cdots g_{j_{k-1}}^{\#}\left(\delta\left(g_{j_{k}}\right)\right), 1\right) * r_{j}\left(\left(1, g_{1}\right), \ldots,\left(1, g_{n}\right)\right)=(1,1) .
$$

In this expression we can explicitly compute $r_{j}\left(\left(1, g_{1}\right), \ldots,\left(1, g_{n}\right)\right)$ as

$$
\left(\prod_{k=2}^{t-1} g_{j_{1}}^{\#} \cdots g_{j_{t-k}}^{\#}\left(\mathcal{F}\left(g_{j_{t-k+1}}, g_{j_{t-k+2}} \cdots g_{j_{t}}\right)\right) \cdot \mathcal{F}\left(g_{j_{1}}, g_{j_{2}} \cdots g_{j_{t}}\right), 1\right) \text {. }
$$

Think of values $\delta\left(g_{i}\right)$ as being variables for the moment. Then each relation (3.2) gives rise to an equation in $N$. It is important to stress out that for the construction of such an equation we only need to know the values $\mathcal{F}\left(g_{j_{k}}, g_{j_{k+1}} \cdots g_{j_{t}}\right)$ and the automorphisms $g_{j_{k}}^{\#}$ for $k=1, \ldots, t-1$. Considering all relators $r_{j} \in R$ thus yields a system of equations, whose solutions correspond to complements. However, solving such a system is rather hopeless in general.

\subsection{Elementary abelian case}

Let us therefore assume that $N$ is an elementary abelian $p$-group of rank $d$. In this case, $N$ can be identify with $d$-dimensional vector space $\mathbb{Z}_{p}^{d}$, the function \# is a homomorphism that defines an action of $G$ on $N$, and the automorphisms $g^{\#}$ of $N$ are invertible $d \times d$ matrices. We search for a complement by considering each $\delta\left(g_{i}\right)$ in $N$ as a vector with variable entries $x_{i, 1}, \ldots, x_{i, d}$. Then each relation gives rise to $d$ linear equations in the variables $x_{i, 1}, \ldots, x_{i, d}$. Putting all together we obtain a non-homogeneous system of $m d$ equations, whose set of all solutions is in bijective correspondence with all the complements. 


\subsection{Solvable case}

The case when $N$ is solvable can be dealt with by choosing a characteristic series

$$
N=N_{0} \triangleright N_{1} \triangleright \cdots \triangleright N_{r}=1
$$

such that each factor $N_{j-1} / N_{j}$ is elementary abelian. The problem reduces into the same problem on $N_{j-1} / N_{j}$ and $N_{j}$ inductively down the series. The following theorem is a first step towards this reduction when the extension $E$ is reconstructed as a crossed product extension $N \operatorname{ext}_{\#, \mathcal{F}} G$.

Theorem 3.1. Let $M, N$ be normal subgroups of a finite group $E$ with $M<N$, and let $G=E / N$.

(i) If $N \operatorname{ext}_{\#, \mathcal{F}} G$ reconstructs $E$, then $N / M \operatorname{ext}_{\#_{N / M}, \mathcal{F}_{N / M}} G$ reconstructs $E / M$ with

$$
\begin{aligned}
g^{\# / M}(n M) & =g^{\#}(n) M \\
\mathcal{F}_{N / M}\left(g_{1}, g_{2}\right) & =\mathcal{F}\left(g_{1}, g_{2}\right) M .
\end{aligned}
$$

(ii) In particular, suppose that $E / M$ splits, and let $L / M$ be a complement of $N / M$ in $E / M$ determined by a function $\delta: G \rightarrow N / M$. Let $T$ be a transversal of $M$ in $N$ and, for each $\delta(g)$, let $\overline{\delta(g)}$ be the representative in $T$ such that $\overline{\delta(g)} M=\delta(g)$. Then $M \operatorname{ext}_{\# \delta}, \mathcal{F}_{\delta} G$ reconstructs $L$ with

$$
\begin{aligned}
g^{\#}(m) & =\overline{\delta(g)} g^{\#}(m) \overline{\delta(g)}-1 \\
\mathcal{F}_{\delta}\left(g_{1}, g_{2}\right) & =\overline{\delta\left(g_{1}\right)} g_{1}^{\#}\left(\overline{\delta\left(g_{2}\right)}\right) \mathcal{F}\left(g_{1}, g_{2}\right){\overline{\delta\left(g_{1} g_{2}\right)}}^{-1} .
\end{aligned}
$$

Proof. Let $M, N \triangleleft E$ with $M<N$, and suppose that $E$ is reconstructed in a form

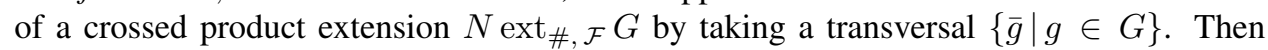
$(E / M) /(N / M) \cong E / N=G$ and $\{\bar{g} M \mid g \in G\}$ is a transversal of $N / M$ in $E / M$. For each $g \in G$ we have the automorphism $g^{\#_{N / M}}$ of $N / M$ defined by

$$
g^{\# / M}(n M)=\bar{g} M n M \bar{g}^{-1} M=\bar{g} n \bar{g}^{-1} M=g^{\#}(n) M,
$$

and hence the weak action $\#_{N / M}: G \rightarrow \operatorname{Aut}(N / M)$ is given by $\#_{N / M}: g \mapsto g^{\#_{N / M}}$. Furthermore,

$$
\overline{g_{1}} M \overline{g_{2}} M{\overline{g_{1} g_{1}}}^{-1} M=\overline{g_{1}} \bar{g}_{2}{\overline{g_{1} g_{2}}}^{-1} M=\mathcal{F}\left(g_{1}, g_{2}\right) M
$$

shows that the factor set $\mathcal{F}_{N / M}: G \times G \rightarrow N / M$ is given by

$$
\mathcal{F}_{N / M}:\left(g_{1}, g_{2}\right) \mapsto \mathcal{F}\left(g_{1}, g_{2}\right) M
$$

This proves (i).

As for (ii), let $L / M$ be a complement of $N / M$ in $E / M$ determined by $\delta: G \rightarrow N / M$; that is, $L / M$ has the form $\{\delta(g) \bar{g} M, \mid g \in G\}$. Fix a transversal $T$ of $M$ in $N$. For each $\delta(g)$ in $N / M$ choose the representative $\overline{\delta(g)}$ in $T$ such that $\overline{\delta(g)} M=\delta(g)$. Then $\{\overline{\delta(g)} \bar{g} \mid g \in G\}$ is a transversal of $M$ in $L$. For $g \in G$ the corresponding automorphism $g^{\# \delta}$ of $M$ is defined by

$$
g^{\# \delta}(m)=\overline{\delta(g)} \bar{g} m \bar{g}^{-1} \overline{\delta(g)}^{-1}=\overline{\delta(g)}^{\#}(m) \overline{\delta(g)}^{-1} .
$$


Hence the weak action $\#_{\delta}: G \rightarrow \operatorname{Aut}(M)$ is given by $\#_{\delta}: g \mapsto g^{\# \delta}$. It remains to compute the corresponding factor set. We have

$$
\begin{aligned}
\overline{\delta\left(g_{1}\right)} \overline{g_{1}} \overline{\delta\left(g_{2}\right)} \overline{g_{2}}\left(\overline{\delta\left(g_{1} g_{2}\right)} \overline{g_{1} g_{2}}\right)^{-1} & =\overline{\delta\left(g_{1}\right)} \overline{g_{1}} \overline{\delta\left(g_{2}\right)} \overline{g_{2}} \overline{g_{1} g_{2}}-1 \overline{\delta\left(g_{1} g_{2}\right)}-1 \\
& =\overline{\delta\left(g_{1}\right)} \overline{g_{1}} \overline{\delta\left(g_{2}\right)} \overline{g_{1}}-1 \overline{g_{1}} \overline{g_{2}} \overline{g_{1} g_{2}}-1 \overline{\delta\left(g_{1} g_{2}\right)}-1 \\
& =\overline{\delta\left(g_{1}\right)} g_{1}^{\#}\left(\overline{\delta\left(g_{2}\right)}\right) \mathcal{F}\left(g_{1}, g_{2}\right) \overline{\delta\left(g_{1} g_{2}\right)}-1
\end{aligned}
$$

and so $\mathcal{F}_{\delta}: G \times G \rightarrow M$ is given by

$$
\mathcal{F}_{\delta}:\left(g_{1}, g_{2}\right) \mapsto \overline{\delta\left(g_{1}\right)} g_{1}^{\#}\left(\overline{\delta\left(g_{2}\right)}\right) \mathcal{F}\left(g_{1}, g_{2}\right){\overline{\delta\left(g_{1} g_{2}\right)}}^{-1}
$$

This completes the proof.

To start the reduction we first need to test whether $E / N_{1}$ is a split extension of $N / N_{1}$ by $G$. By Theorem 3.1(i) we reconstruct $E / N_{1}$ in a form of a crossed product extension

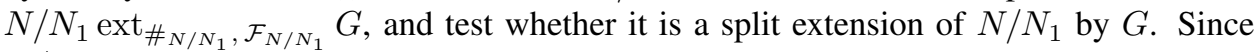
$N / N_{1}$ is elementary abelian, this is done by solving a non-homogeneous system of linear equations described in Subsection 3.1. If the system has no solution, then $E$ does not split. Otherwise, each solution $\delta$ uniquely determines a complement $L / N_{1}$ of $N / N_{1}$ in $E / N_{1}$. We further need to test each $L$ (corresponding to each $\delta$ ) for being a split extension of $N_{1}$ by $G$. Using Theorem 3.1(ii) we reconstruct each such $L$ in a form of a crossed product extension $N_{1} \operatorname{ext}_{\#_{\delta}}, \mathcal{F}_{\delta} G$, and continue down the series.

Suppose inductively that, for some $j<r$, we have complements $\bar{L} / N_{j}$ of $N / N_{j}$ in $E / N_{j}$, and that each $\bar{L}$ is reconstructed as a crossed product $N_{j} \operatorname{ext}_{\overline{\#}, \overline{\mathcal{F}}} G$. In order to test whether each such $\bar{L} / N_{j+1}$ is a split extension of $N_{j} / N_{j+1}$ by $G$ we reconstruct $\bar{L} / N_{j+1}$ in a form

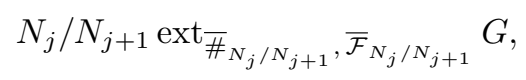

and test whether the latter is a split extension of $N_{j} / N_{j+1}$ by $G$. Again, $N_{j} / N_{j+1}$ is elementary abelian, so we need to solve an appropriate linear system. If none of $\bar{L} / N_{j+1}$ are split extensions, then neither is $E$. Otherwise, for each $\bar{L} / N_{j+1}$ that splits, solutions $\delta^{*}$ uniquely determine complements $L^{*} / N_{j+1}$ of $N_{j} / N_{j+1}$ in $\bar{L} / N_{j+1}$. Clearly, each $L^{*} / N_{j+1}$ is also a complement of $N / N_{j+1}$ in $E / N_{j+1}$. Finally, we reconstruct each $L^{*}$ in a form $N_{j+1} \operatorname{ext}_{\overline{\#}_{\delta^{*}}, \overline{\mathcal{F}}_{\delta^{*}}} G$, and proceed to the next step.

Observe that at each step it is enough to consider complements only up to conjugacy. Reduction up to conjugacy can be described by an action on the set of solutions $\delta^{*}$ that determine complements, see [3] and [8, Chapter 8] for more details.

\section{An improved algorithm for testing whether the lifted group splits}

The general method described in Section 3 will be now applied in the context of lifting automorphisms along regular covering projections.

Let $\zeta: A(X) \rightarrow N$ be a voltage function associated with a solvable regular covering projection $\wp: \tilde{X} \rightarrow X$ of connected graphs, and let $G \leq \operatorname{Aut}(X)$ lift to $\tilde{G}$. We derive an algorithm for testing whether the lifted group $\tilde{G}$ is a split extension of $\mathrm{CT}(\wp)$ by $G$. In contrast with [17] we avoid the combinatorial reconstruction not only of the covering 
graph $\tilde{X}$ and the lifted group $\tilde{G}$, but also of the all intermediate elementary abelian regular covering projections $\wp_{j}: X_{j} \rightarrow X_{j-1}$ in the decomposition

$$
\tilde{X}=X_{n} \stackrel{\wp_{n}}{\rightarrow} X_{n-1} \rightarrow \cdots \rightarrow X_{1} \stackrel{\wp_{1}}{\rightarrow} X_{0}=X
$$

of $\wp$ arising from a characteristic series $N=N_{0} \triangleright N_{1} \triangleright \cdots \triangleright N_{r}=1$ with elementary abelian factors $N_{j-1} / N_{j}$. Consequently, we neither reconstruct the graphs $X_{j}$ nor the intermediate complements acting on $X_{j}$.

Instead, we first reconstruct $\tilde{G}$ in a form of a crossed product extension $N \operatorname{ext}_{\#, \mathcal{F}} G$ derived from the voltage function $\zeta: A(X) \rightarrow N$. Recall from Preliminaries that, since $G$ lifts, for each $g \in G$, there exists an automorphism $g^{\# v}$ of $N$ uniquely determined by a map $\zeta W^{*} \mapsto \zeta g\left(W^{*}\right)$, where $W^{*}$ ranges over all fundamental closed walks in $X$ rooted at $v$. As it is proved in [15], choosing a base vertex $v$, the function \#: $G \rightarrow \operatorname{Aut}(N)$, given by

$$
\#: g \mapsto g^{\# v},
$$

is in fact the weak action, while the factor set $\mathcal{F}: G \times G \rightarrow N$ is given by

$$
\mathcal{F}:\left(g_{1}, g_{2}\right) \mapsto g_{1}^{\# v}(\zeta Q)\left(\zeta g_{1}(Q)\right)^{-1}, \quad \text { for a walk } Q \text { from } g_{2}(v) \text { to } v \text {. }
$$

In view of the approach in Section 3, if $G$ has a presentation $\langle S \mid R\rangle$ we actually only need to know the automorphisms $g_{i}^{\# v}$ for all $g_{i} \in S$ and, for each $r_{j}=g_{j_{1}} \cdots g_{j_{t}} \in R$, the values $\mathcal{F}\left(g_{j_{k}}, g_{j_{k+1}} \cdots g_{j_{t}}\right)$ for $k=1, \ldots, t-1$. As each $g_{i}^{\# v}$ is uniquely determined by $\zeta W^{*} \mapsto \zeta g_{i}\left(W^{*}\right)$, we only store the voltages $\zeta W^{*}$ of the fundamental closed walks $W^{*}$ at $v$ together with the voltages $\zeta g_{i}\left(W^{*}\right)$ of the mapped walks. All these data can be efficiently computed, for instance, by using breadth first search on $X$ that starts at root $v$. Finally, with these data in hand we simply follow the approach described in Subsection 3.2.

\section{Performance}

In order to verify the effectiveness of the proposed algorithm we compare its performance with the previous version (called ISA, see [17]). The new version, called ISAI from now on, has been implemented in MAGMA. The source code of both versions is available online [18].

A test has been performed on a subset of the database described in [17]. In particular, we have selected solvable regular covering projections for the complete graph $K_{5}$, the Petersen graph $G P(5,2)$, the Ljubljana graph $\mathcal{L}$ [4], and the graph F258A [2] along which the full automorphism group lifts. Elementary abelian coverings have been eliminated since ISAI actually coincides with ISA on such coverings. Both algorithms were run on an $2.93 \mathrm{GHz}$ Quad-Core Intel ${ }^{\circledR}$ Xeon ${ }^{\circledR}$ processor X7350 at the Faculty of Mathematics and Physics, University of Ljubljana.

Results are gathered in Tables 1-4. The first column shows the order of the covering graph, while the second one describes the type of the voltage group: solvable, but not abelian; or, abelian, but not elementary abelian. Further, the notation used in the third column for identifying the voltage group is the library number in the database of small groups in MAGMA. Execution times given in seconds (CPU time) are displayed in the fourth and the fifth column (for ISA and ISAI, respectively). The last column indicates whether the corresponding lift of the full automorphism group splits. As can be seen from results, ISAI is clear winner of the comparison. 
Table 1: Performance comparison for the complete graph $K_{5}$

\begin{tabular}{cccccc}
\hline \hline $\begin{array}{c}\text { Order of covering } \\
\text { graph }\end{array}$ & $\begin{array}{c}\text { Type of voltage } \\
\text { group }\end{array}$ & $\begin{array}{c}\text { Library number of } \\
\text { voltage group }\end{array}$ & $t_{\text {ISA }}(s)$ & $t_{\text {ISAI }}(s)$ & Split? \\
\hline 30 & Solvable & $\langle 6,1\rangle$ & 0.010 & 0.010 & true \\
120 & Solvable & $\langle 24,12\rangle$ & 0.050 & 0.040 & true \\
240 & Solvable & $\langle 48,28\rangle$ & 0.520 & 0.090 & false \\
480 & Solvable & $\langle 96,230\rangle$ & 0.350 & 0.040 & true \\
640 & Solvable & $\langle 128,2326\rangle$ & 1.530 & 0.050 & true \\
960 & Solvable & $\langle 192,1542\rangle$ & 1.530 & 0.060 & true \\
1250 & Abelian & $\langle 250,15\rangle$ & 0.020 & 0.050 & false \\
1280 & Solvable & $\langle 256,55642\rangle$ & 1.670 & 0.070 & true \\
\hline
\end{tabular}

Table 2: Performance comparison for the Petersen graph

\begin{tabular}{cccccc}
\hline \hline $\begin{array}{c}\text { Order of covering } \\
\text { graph }\end{array}$ & $\begin{array}{c}\text { Type of voltage } \\
\text { group }\end{array}$ & $\begin{array}{c}\text { Library number of } \\
\text { voltage group }\end{array}$ & $t_{\text {ISA }}(s)$ & $t_{\text {ISAI }}(s)$ & Split? \\
\hline 80 & Solvable & $\langle 8,4\rangle$ & 0.020 & 0.060 & false \\
360 & Solvable & $\langle 36,10\rangle$ & 0.020 & 0.020 & true \\
720 & Solvable & $\langle 72,24\rangle$ & 0.020 & 0.020 & false \\
1080 & Solvable & $\langle 108,17\rangle$ & 0.610 & 0.040 & true \\
1280 & Solvable & $\langle 128,2321\rangle$ & 1.770 & 0.020 & false \\
1620 & Solvable & $\langle 162,54\rangle$ & 0.020 & 0.020 & true \\
2160 & Solvable & $\langle 216,33\rangle$ & 0.030 & 0.030 & false \\
2500 & Abelian & $\langle 250,15\rangle$ & 0.030 & 0.030 & false \\
2560 & Solvable & $\langle 256,55628\rangle$ & 1.810 & 0.030 & false \\
\hline
\end{tabular}

Table 3: Performance comparison for the Ljubljana graph $\mathcal{L}$

\begin{tabular}{cccccc}
\hline \hline $\begin{array}{c}\text { Order of covering } \\
\text { graph }\end{array}$ & $\begin{array}{c}\text { Type of voltage } \\
\text { group }\end{array}$ & $\begin{array}{c}\text { Library number of } \\
\text { voltage group }\end{array}$ & $t_{\text {ISA }}(s)$ & $t_{\text {ISAI }}(s)$ & Split? \\
\hline 896 & Solvable & $\langle 8,4\rangle$ & 0.650 & 0.030 & true \\
1344 & Solvable & $\langle 12,3\rangle$ & 0.560 & 0.040 & true \\
1792 & Abelian & $\langle 16,2\rangle$ & 0.630 & 0.030 & true \\
2352 & Solvable & $\langle 21,1\rangle$ & 0.600 & 0.030 & true \\
2688 & Solvable & $\langle 24,11\rangle$ & 3.090 & 0.040 & true \\
\hline
\end{tabular}

Table 4: Performance comparison for the graph F258A

\begin{tabular}{cccccc}
\hline \hline $\begin{array}{c}\text { Order of covering } \\
\text { graph }\end{array}$ & $\begin{array}{c}\text { Type of voltage } \\
\text { group }\end{array}$ & $\begin{array}{c}\text { Library number of } \\
\text { voltage group }\end{array}$ & $t_{\text {ISA }}(s)$ & $t_{\text {ISAI }}(s)$ & Split? \\
\hline \multirow{2}{*}{2064} & Solvable & $\langle 8,4\rangle$ & 2.660 & 0.120 & true \\
3096 & Abelian & $\langle 12,5\rangle$ & 2.720 & 0.150 & false \\
4128 & Abelian & $\langle 16,2\rangle$ & 2.670 & 0.130 & true \\
\hline
\end{tabular}

Acknowledgement. The author would like to thank Aleksander Malnič for enlightening discussions. 


\section{References}

[1] W. Bosma, J. Cannon, C. Playoust, The Magma algebra system I: The user language, J. Symb. Comput. 24 (1997), 235-265.

[2] I. Z. Bouwer (ed.), The Foster Census, Charles Babbage Research Centre, Winnipeg, 1988.

[3] F. Celler, J. Neubser, C. R.B. Wright, Some remarks on the computation of complements and normalizers in solvable groups, Acta Applicandae Mathematicae 21 (1990), 57-76.

[4] M. D. E. Conder, A. Malnič, D. Marušič, T. Pisanski, P. Potočnik, The cubic edge-but not vertex-transitive graph on 112 vertices, J. Graph Theory 50 (2005), 25-42.

[5] M. D. E. Conder, A. Malnič, D. Marušič, P. Potočnik, A census of cubic semisymmetric graphs on up to 768 vertices, J. Algebraic Combin. 23 (2006), 255-294.

[6] S. F. Du, J. H. Kwak, M. Y. Xu, 2-arc-transitive regular covers of complete graphs having the covering transformation group $\mathbb{Z}_{p}^{3}$, J. Combin. Theory Ser. B 93 (2005), 73-93.

[7] J. L. Gross, T. W. Tucker, Topological Graph Theory, Wiley - Interscience, New York, 1987.

[8] D. Holt, B. Eick, E. A. O'Brien, Handbook of Computational Group Theory, Chapman and Hall/CRC, Boca Raton London New York Washington D.C., 2005.

[9] M. Knor, P. Potočnik, Efficient domination in cubic vertex-transitive graphs, European J. Combin. 33 (2012), 1755-1764.

[10] I. Kovács, K. Kutnar, D. Marušič, Classification of edge-transitive rose window graphs, J. Graph Theory 65 (2010), 216-231.

[11] S. McLane Homology, Springer-Verlag, Berlin Gottingen Heidelberg, 1963.

[12] A. Malnič, Group actions, coverings and lifts of automorphisms, Discrete Math. 182 (1998), 203-218.

[13] A. Malnič, D. Marušič, P. Potočnik, On cubic graphs admitting an edge-transitive solvable group, J. Algebraic Combin. 20 (2004), 99-113.

[14] A. Malnič, R. Nedela, M. Škoviera, Lifting graph automorphisms by voltage assignments, European J. Combin. 21 (2000), 927-947.

[15] A. Malnič, R. Požar, On the Split Structure of Lifted Groups, Ars Math. Contemp. 10 (2016) 113-134.

[16] P. Potočnik, Edge-colourings of cubic graphs admitting a solvable vertex-transitive group of automorphisms, J. Combin. Theory Ser. B 91 (2004), 289-300.

[17] R. Požar, Some computational aspects of solvable regular covers of graphs, J. Symb. Comput. 70 (2015), 1-13.

[18] R. Požar, http://osebje.famnit.upr.si/ rok.pozar.

[19] J. X. Zhou, Y. Q. Feng, Semisymmetric elementary abelian covers of the Heawood graph, Discrete Math. 310 (2010), 3658-3662. 